Pacific Journal of Mathematics

ISOMETRIES OF $C^{(n)}[0,1]$ 


\section{ISOMETRIES OF $C^{(n)}[0,1]$}

\section{D. PATHAK}

By $C^{(n)}[0,1]$ (henceforth denoted by $C^{(n)}$ ) we denote the Banach algebra of complex valued $n$ times continuously differentiable functions on $[0,1]$ with norm given by

$$
\|f\|=\sup _{x \in[0,1]}\left(\sum_{r=0}^{n}\left(\frac{\left|f^{(r)}(x)\right|}{r !}\right) \text { for } f \in C^{(n)}\right. \text {. }
$$

By an isometry of $C^{(n)}$ we mean a norm-preserving linear map of $C^{(n)}$ onto itself.

The purpose of this article is to describe the isometries of $C^{(n)}$ for any positive integer $n$. More precisely, we show that any isometry of $C^{(n)}$ is induced by a point map of the interval $[0,1]$ onto itself.

The isometries of $C^{(1)}$ (with the same norm as above) are determined by M. Cambern [1]. N. V. Rao and A. K. Roy [2] have also determined the isometries of $C^{(1)}$ with norm of $f \in C^{(1)}$ given by $\|f\|=\|f\|_{\infty}+\left\|f^{\prime}\right\|_{\infty}$ and even for more general norms.

In the proof we shall follow the techniques of [1].

1. Let $W$ denote the compact space $[0,1] \times[-\pi, \pi]^{n}$. We prove the following propositions.

Proposition 1.1. Given $\left(x, \theta_{1}, \cdots, \theta_{n}\right) \in W$, then there exists $h \in$ $C^{(n)}$ such that

$$
\sum_{r=0}^{n} \frac{\left|h^{(r)}(x)\right|}{r !}>\sum_{r=0}^{n} \frac{\left|h^{(r)}(y)\right|}{r !}
$$

for $y \in[0,1], y \neq x$, with $|h(x)|=h(x)>0,\left|h^{\prime}(x)\right|=e^{i \theta_{1}} h^{\prime}(x)>0$, $\left|h^{\prime \prime}(x)\right|=e^{i \theta_{2}} h^{\prime \prime}(x)>0, \cdots,\left|h^{(n)}(x)\right|=e^{i \theta_{n}} h^{(n)}(x)>0$.

Proof. Let $f_{0}$ be the real valued, nonnegative continuous function on $[0,1]$ defined as follows

$$
f_{0}(y)= \begin{cases}0 & \cdots(y-x) \leqq-\frac{1}{2(n !)} \\ 1+2(n !)(y-x) & \cdots-\frac{1}{2(n !}<(y-x) \leqq 0 \\ 1-2(n !)(y-x) & \cdots 0<(y-x) \leqq \frac{1}{2(n !)} \\ 0 & \cdots \frac{1}{2(n !)}<(y-x) .\end{cases}
$$


For $1 \leqq r \leqq n$ define $f_{r}(y)$ as $f_{r}(y)=\int_{x}^{y} f_{r-1}(t) d t$. It can be easily verified that for $1 \leqq r \leqq n, f_{r}(y)$ is as follows:

$$
f_{r}(y)=\left\{\begin{array}{l}
-\sum_{j=1}^{r} \frac{1}{(j+1) !(2(n !))^{j}} \frac{(y-x)^{r-j}}{(r-j) !} \cdots(y-x) \leqq \frac{-1}{2(n !)} \\
\frac{(y-x)^{r}}{r !}+\frac{2(n !)(y-x)^{r+1}}{(r+1) !} \cdots-\frac{1}{2(n !)}<(y-x) \leqq 0 \\
\frac{(y-x)^{r}}{r !}-\frac{2(n !)(y-x)^{r+1}}{(r+1) !} \cdots 0<(y-x) \leqq \frac{1}{2(n !)} \\
\sum_{j=1}^{r} \frac{(-1)^{j-1}}{(j+1) !(2(n !))^{j}} \frac{(y-x)^{r-j}}{(r-j) !} \cdots \frac{1}{2(n !)}<(y-x) .
\end{array}\right.
$$

Now let

$$
g(y)=\frac{1}{(2 n-1) !}\left[\sum_{j=1}^{(n-1)} e^{i\left(\theta_{1}-\theta_{j}\right)} \frac{(y-x)^{j}}{j !}\right]+e^{i\left(\theta_{1}-\theta_{n}\right)} f_{n}(y)
$$

Clearly, for $1 \leqq r \leqq n, f_{n}^{(r)}=f_{n-r}$. Therefore $g \in C^{(n)}$ and

$$
g^{(r)}(y)=\frac{1}{(2 n-1) !} \sum_{j=r}^{(n-1)} e^{i\left(\theta_{1}-\theta_{j}\right)} \frac{(y-x)^{j-r}}{(j-r) !}+e^{i\left(\theta_{1}-\theta_{n}\right)} f_{n-r}(y) \text { for } 1 \leqq r \leqq n
$$

Thus

$$
g(x)=0, g^{(r)}(x)=\frac{1}{(2 n-1) !} e^{i\left(\theta_{1}-\theta_{r}\right)} \text { for } 1 \leqq r \leqq n-1,
$$

and $g^{(n)}(x)=e^{i\left(\theta_{1}-\theta_{n}\right)}$. Therefore

$$
\sum_{r=0}^{n} \frac{\left|g^{(r)}(x)\right|}{r !}=\frac{1}{(2 n-1) !} \sum_{r=1}^{(n-1)} \frac{1}{r !}+\frac{1}{n !} .
$$

Now consider $\sum_{r=0}^{n}\left(\left|g^{(r)}(y)\right| / r !\right)$ for $y \in[0,1]$ and $y \neq x$.

Case 1. Let $(y-x) \leqq(-1 / 2(n !))$.

$$
\begin{aligned}
\sum_{r=0}^{n} \frac{\left|g^{(r)}(y)\right|}{r !} \leqq \frac{1}{(2 n-1) !} \sum_{j=1}^{(n-1)} \frac{|y-x|^{j}}{j !}+\frac{1}{(2 n-1) !} \sum_{r=1}^{(n-1)} \frac{1}{r !} \\
\quad \times\left\{\sum_{j=r}^{(n-1)} \frac{|y-x|^{j-r}}{(j-r) !}\right\}+\sum_{r=0}^{n} \frac{1}{r !}\left\{\sum_{j=1}^{(n-r)} \frac{|y-x|^{(n-r-j)}}{(j+1) !(2(n !))^{j}(n-r-j) !}\right\} .
\end{aligned}
$$

For $n=1,2$, it can be easily verified that right hand side of (1) is less than $\sum_{r=0}^{n}\left(\left|g^{(r)}(x)\right| / r !\right)$. When $n \geqq 3$, denoting $(n ! /(n-j) ! j !)$ by $C_{j}^{n}$, (1) gives 


$$
\begin{aligned}
\sum_{r=0}^{n} \frac{\left|g^{(r)}(y)\right|}{r !} \leqq & \frac{1}{(2 n-1) !} \sum_{j=1}^{(n-1)} \frac{1}{j !}+\frac{1}{(2 n-1) !} \sum_{r=1}^{(n-1)} \frac{(n-1)}{r !} \\
& +\frac{1}{2(n !)} \sum_{r=0}^{(n-1)} \frac{1}{r !} \sum_{j=1}^{(n-r)}\left\{\frac{1}{j(j+1)(n-r-1) !} C_{j-1}^{n-r-1} \frac{1}{(2(n !))^{j-1}}\right\} .
\end{aligned}
$$

Now

$$
\begin{array}{r}
\frac{1}{(2 n-1) !} \sum_{r=1}^{(n-1)} \frac{(n-1)}{r !} \leqq \frac{(n-1)}{(2 n-1) !} \sum_{r=1}^{(n-1)} \frac{1}{2^{r-1}}<\frac{2(n-1)}{(2 n-1) !}<\frac{1}{4(n !)} \\
\quad \text { for all } n \geqq 3 .
\end{array}
$$

Thus we have

$$
\text { for } n \geqq 3, \frac{1}{(2 n-1) !} \sum_{r=1}^{(n-1)} \frac{(n-1)}{r !}<\frac{1}{4(n !)} .
$$

Also

$$
\begin{aligned}
\frac{1}{2(n !)} \sum_{r=0}^{(n-1)} \frac{1}{r !} \sum_{j=1}^{(n-r)}\left\{\frac{1}{j(j+1)(n-r-1) !} C_{j-1}^{n-r-1} \frac{1}{(2(n !))^{j-1}}\right\} \\
\leqq \frac{1}{2(n !)} \sum_{r=0}^{(n-1)} \frac{1}{r ! 2(n-r-1) !}\left\{\sum_{j=1}^{(n-r)} C_{j-1}^{n-r-1} \frac{1}{(2(n !))^{j-1}}\right\} \\
=\frac{1}{(2(n !))} \cdot \frac{1}{2(n-1) !} \sum_{r=0}^{(n-1)} C_{r}^{n-1}\left(1+\frac{1}{2(n !)}\right)^{(n-1-r)} \\
=\frac{\left\{\left(1+\frac{1}{2(n !)}\right)+1\right\}^{n-1}}{2(2(n !))(n-1) !} \leqq \frac{\left(\frac{9}{4}\right)^{n-1}}{2(2(n !))(n-1) !}<\frac{81}{64} \cdot \frac{1}{2(n !)} .
\end{aligned}
$$

Thus

$$
\begin{aligned}
& \frac{1}{2(n !)} \sum_{r=0}^{(n-1)} \frac{1}{r !} \sum_{j=1}^{(n-r)}\left\{\frac{1}{j(j+1)(n-r-1) !} \cdot C_{j-1}^{n-r-1} \cdot \frac{1}{(2(n !))^{j-1}}\right\} \\
& \quad<\frac{3}{4} \cdot \frac{1}{n !} .
\end{aligned}
$$

By (2) and (3) it follows immediately that for all $y \in[0,1]$ and $y \neq x$

$$
\sum_{r=0}^{n} \frac{\left|g^{(r)}(y)\right|}{r !}<\sum_{r=0}^{n} \frac{\left|g^{(r)}(x)\right|}{r !} .
$$

Case 2. Let $-(1 / 2(n !))<(y-x)<0$

$$
\begin{aligned}
\sum_{r=0}^{n} \frac{\left|g^{(r)}(y)\right|}{r !} \leqq & \frac{1}{(2 n-1) !} \sum_{j=1}^{(n-1)} \frac{|y-x|^{j}}{j !}+\frac{1}{(2 n-1) !} \sum_{r=1}^{(n-1)} \frac{1}{r !}\left\{\sum_{j=r}^{(n-1)} \frac{|y-x|^{j-r}}{(j-r) !}\right\} \\
& +\sum_{r=0}^{n} \frac{1}{r !}\left|\frac{(y-x)^{n-r}}{(n-r) !}+\frac{2(n !)(y-x)^{n-r+1}}{(n-r+1) !}\right|
\end{aligned}
$$




$$
\begin{aligned}
= & \frac{1}{(2 n-1) !} \sum_{j=1}^{(n-1)} \frac{(-1)^{j}(y-x)^{j}}{j !}+\frac{1}{(2 n-1) !} \sum_{r=1}^{n-1} \frac{1}{r !} \\
& +\frac{1}{(2 n-1) !} \sum_{r=1}^{(n-2)} \frac{1}{r !}\left\{\sum_{j=r+1}^{(n-1)} \frac{(-1)^{j-r}(y-x)^{j-r}}{(j-r) !}\right\} \\
& +\sum_{r=0}^{n} \frac{(-1)^{n-r}}{r !}\left\{\frac{(y-x)^{n-r}}{(n-r) !}+\frac{2(n !)(y-x)^{n-r+1}}{(n-r+1) !}\right\} \\
= & \frac{1}{(2 n-1) !} \sum_{r=1}^{(n-1)} \frac{1}{r !}+\frac{1}{n !}+\sum_{s=1}^{(n-1)}(y-x)^{s}\left\{\frac{(-1)^{s}}{s !(2 n-1) !}\right. \\
& \left.+\frac{(-1)^{s}}{s !(n-s) !}+\frac{2(n !)(-1)^{s-1}}{s !(n-s+1) !}+\sum_{r=1}^{n-1-s} \frac{(-1)^{s}}{s ! r !}\right\} \\
& +(y-x)^{n}\left\{\frac{(-1)^{n}}{n !}+\frac{2(n !)(-1)^{n-1}}{n !}\right\}+\frac{(-1)^{n}(y-x)^{n+1}}{(n+1) !} \\
= & \sum_{r=0}^{n} \frac{\left|g^{(r)}(x)\right|}{r !}+\sum_{s=1}^{(n-1)} \frac{(-1)^{s}(y-x)^{s}}{s !}\left\{\frac{1}{(2 n-1) !}\right. \\
& \left.+\sum_{r=1}^{(n-s)} \frac{1}{r !}-\frac{2(n !)}{(n-s+1) !}\right\} \\
& +\frac{(-1)^{n}(y-x)^{n}}{n !}\{1-2(n !)\}+\frac{(-1)^{n}(y-x)^{n+1}}{(n+1) !} \\
< & \sum_{r=0}^{n} \frac{\left|g^{(r)}(x)\right|}{r !}
\end{aligned}
$$

since all the other terms are negative. Verification in cases when $0<(y-x) \leqq(1 / 2(n !))$ and $(1 / 2(n !))<(y-x)$ is similar. From this it follows that the function $h \in C^{(n)}$ defined by $h(y)=1+e^{-i \theta_{1}} g(y)$ has the desired properties.

Proposition 1.2. For any $f \in C^{(n)}$

$$
\sum_{j=1}^{n}(-1)^{j-1} C_{j-1}^{n}\left(f^{n-j+1}\right)^{(k)}(x)(f(x))^{j-1}= \begin{cases}0 & \text { if } 1 \leqq k<n \\ n !\left(f^{\prime}(x)\right)^{n} & \text { if } k=n\end{cases}
$$

where $\left(f^{n-j+1}\right)^{(k)}(x)$ means the $k$ th derivative of $f^{n-j+1}$ at $x$.

Proof. We prove this proposition by induction on $n$. For $n=1$ it is obvious. Let it be true for $n=r$. Then we have

$$
\sum_{j=1}^{r}(-1)^{j-1} C_{j-1}^{r}\left(f^{r-j+1}\right)^{(k)}(x)(f(x))^{j-1}=0, \quad \text { for } 1 \leqq k<r,
$$

and

$$
\sum_{j=1}^{r}(-1)^{j-1} C_{j-1}^{r}\left(f^{r-j+1}\right)^{(r)}(x)(f(x))^{j-1}=r !\left(f^{\prime}(x)\right)^{r} .
$$

Now let $n=r+1$ and $k=r+1$. 
Since $\left(f^{r-j+2}\right)^{\prime}(x)=(r-j+2)\left(f^{r-j+1}\right)(x) f^{\prime}(x)$

$$
\begin{aligned}
\sum_{j=1}^{(r+1)}(- & 1)^{j-1} C_{j-1}^{r+1}\left(f^{r-j+2}\right)^{(r+1)}(x)(f(x))^{j-1} \\
= & \sum_{j=1}^{r+1}(-1)^{j-1} C_{j-1}^{r+1}(f(x))^{j-1}\left\{(r-j+2) \sum_{s=0}^{r} C_{s}^{r}\left(f^{r-j+1}\right)^{(r-s)}(x)\left(f^{\prime}\right)^{(s)}(x)\right\} \\
= & \sum_{j=1}^{r+1}(-1)^{j-1}(r+1) C_{j-1}^{r}(f(x))^{j-1}\left(f^{r-j+1}\right)^{(r)}(x) f^{\prime}(x) \\
& +\sum_{j=1}^{r+1}(-1)^{j-1}(r+1) C_{j-1}^{r}(f(x))^{j-1}\left\{\sum_{s=1}^{r} C_{s}^{r}\left(f^{r-j+1}\right)^{(r-s)}(x)\left(f^{\prime}\right)^{(s)}(x)\right\} \\
= & (r+1)\left\{\sum_{j=1}^{r}(-1)^{j-1} C_{j-1}^{r}(f(x))^{j-1}\left(f^{r-j+1}\right)^{(r)}(x)\right\} f^{\prime}(x) \\
& +(r+1) \sum_{j=1}^{r+1}(-1)^{j-1}(f(x))^{j-1} C_{j-1}^{r}\left\{\sum_{s=1}^{r} c_{s}^{r}\left(f^{r-j+1}\right)^{(r-s)}(x)\left(f^{\prime}\right)^{(s)}(x)\right\} \\
= & (r+1) !\left(f^{\prime}(x)\right)^{r+1}+(r+1) \sum_{s=1}^{r-1} C_{s}^{r}\left(f^{\prime}\right)^{(s)}(x) \\
& \times\left\{\sum_{j=1}^{r}(-1)^{j} C_{j-1}^{r}\left(f^{r-j+1}\right)^{(r-s)}(x)(f(x))^{j-1}\right\} \\
& +(r+1) \sum_{j=1}^{r+1}(-1)^{j-1} C_{j-1}^{r}(f(x))^{r}\left(f^{\prime}(x)\right)^{(r)} \\
= & (r+1) !\left(f^{\prime}(x)\right)^{r+1} .
\end{aligned}
$$

Now let $n=r+1$ and $k<(r+1)$. Then

$$
\begin{aligned}
\sum_{j=1}^{r+1}(- & 1)^{j-1} C_{j-1}^{r+1}\left(f^{r-j+2}\right)^{(k)}(x)(f(x))^{j-1} \\
= & \sum_{j=1}^{r+1}(-1)^{j-1} C_{j-1}^{r+1}(r-j+2)(f(x))^{j-1}\left\{\sum_{s=0}^{k-1} C_{s}^{k-1}\left(f^{r-j+1}\right)^{k-1-s}(x)\left(f^{\prime}\right)^{(s)}(x)\right\} \\
= & (r+1) \sum_{s=0}^{k-2} C_{s}^{k-1}\left(f^{\prime}\right)^{(s)}(x)\left\{\sum_{j=1}^{r}(-1)^{j-1} C_{j-1}^{r}(f(x))^{j-1}\left(f^{r-j+1}\right)^{(k-1-s)}(x)\right\} \\
& +(r+1) \sum_{j=1}^{r+1}(-1)^{j-1} C_{j-1}^{r}(f(x))^{k-1}\left(f^{\prime}\right)^{(k-1)}(x) \\
= & 0 .
\end{aligned}
$$

Hence the proposition follows by mathematical induction.

2. If $X$ is any compact Hausdorff space, we will denote by $C(X)$ the Banach algebra of continuous complex functions defined on $X$ with norm \|\|$_{\infty}$ determined by $\|g\|_{\infty}=\sup _{x \in X}|g(x)|$ for $g \in C(X)$. Given $f \in C^{(n)}$, we define $\tilde{f} \in C(W)$ by

$$
\begin{array}{r}
\tilde{f}\left(x, \theta_{1}, \cdots, \theta_{n}\right)=f(x)+e^{i \theta_{1}} f^{\prime}(x)+\frac{e^{i \theta_{2}}}{2 !} f^{\prime \prime}(x)+\cdots+\frac{e^{i \theta_{n}}}{n !} f^{(n)}(x), \\
\left(x, \theta_{1}, \cdots, \theta_{n}\right) \in W .
\end{array}
$$

The following lemma is then obvious. 
Lemma 2.1. The mapping $f \rightarrow \widetilde{f}$ establishes a linear and normpreserving correspondence between $C^{(n)}$ and the closed subspace $S$ of $C(W), S=\left\{\tilde{f}: f \in C^{(n)}\right\}$.

Next given $\left(x, \theta_{1}, \cdots, \theta_{n}\right) \in W$, we define a continuous linear functional $L\left(x, \theta_{1}, \cdots, \theta_{n}\right)$ on $C^{(n)}$ by

$$
L_{\left(x, \theta_{1}, \ldots, \theta_{n}\right)}(f)=\widetilde{f}\left(x, \theta_{1}, \cdots, \theta_{n}\right), \quad f \in C^{(n)} .
$$

In view of Proposition 1.1 the proof of the following lemma is analogous to the proof of Lemma 1.2 in [1].

LeMma 2.2. An element of $C^{(n) *}$ is an extreme point of the unit ball $U^{*}$ of $C^{(n)^{*}}$ if and only if $f^{*}$ is of the form $e^{i \eta} L_{\left(x, \theta_{1}, \ldots, \theta_{n}\right)}$ for some $\eta \in[-\pi, \pi],\left(x, \theta_{1}, \cdots, \theta_{n}\right) \in W$.

We now suppose that $T$ is an isometry of $C^{(n)}$. The adjoint $T^{*}$ is then an isometry of $C^{(n)^{*}}$, and thus carries extreme points of $U^{*}$ onto itself.

Lemma 2.3. The image by $T$ of the constant function 1 of $C^{(n)}$ is a constant function $e^{i \lambda}, \lambda \in[-\pi, \pi]$.

Proof. For each extreme point $e^{i \eta} L_{\left(x, \theta_{1}, \cdots, \theta_{n}\right)}$ of $U^{*}$,

$$
\left|\left(e^{i \eta} L_{\left(x, \theta_{1}, \cdots, o_{n}\right)}\right)(1)\right|=1 \text {. }
$$

Thus for each extreme point $\left|T^{*}\left(e^{i \eta} L_{\left(x, \theta_{1}, \ldots, \theta_{n}\right)}\right)(1)\right|=1$. Therefore, $\left|L_{\left(x, \theta_{1}, \cdots, \theta_{n}\right)}(T(1))\right|=1$. Thus for a fixed $x, \mid(T(1))(x)+e^{i \theta_{1}}(T(1))^{\prime}(x)+$ $\cdots+\left(e^{i \theta_{n}} / n !\right)(T(1))^{(n)}(x) \mid=1$ for all $\left(\theta_{1}, \cdots, \theta_{n}\right) \in[-\pi, \pi]^{n}$. Choosing $\theta_{1}, \theta_{2}, \cdots, \theta_{n}$, so that

$$
\arg ((T(1))(x))=\arg \left(e^{i \theta_{1}}(T(1))^{\prime}(x)\right)=\cdots=\arg \left(\frac{e^{i \theta_{n}}}{n !}(T(1))^{(n)}(x)\right)
$$

we get

$$
|(T(1))(x)|+\left|(T(1))^{\prime}(x)\right|+\cdots+\frac{\left|(T(1))^{(n)}(x)\right|}{n !}=1 .
$$

Again by choosing $\theta_{1}, \cdots, \theta_{n}$, so that

$$
\arg ((T(1))(x))=\pi+\arg \left(e^{i \theta_{1}}(T(1))^{\prime}(x)\right)=\cdots=\pi+\arg \left(e^{i \theta_{n}}(T(1))^{(x)}(x)\right)
$$

we get

$$
||(T(1))(x)\left|-\left\{\left|(T(1))^{\prime}(x)\right|+\cdots+\frac{\left|(T(1))^{(n)}(x)\right|}{n !}\right\}\right|=1 .
$$

Thus either 


$$
\left\{|(T(1))(x)|=1 \quad \text { and } \quad\left|(T(1))^{\prime}(x)\right|+\cdots+\frac{\left|(T(1))^{(n)}(x)\right|}{n !}=0\right\}
$$

or

(4) $\quad\left\{|(T(1))(x)|=0\right.$ and $\left.\left|(T(1))^{\prime}(x)\right|+\cdots+\frac{\left|(T(1))^{(n)}(x)\right|}{n !}=1\right\}$.

Therefore, for any $x \in[0,1],|(T(1))(x)|=1$ or $|(T(1))(x)|=0$. But since $|T(1)|$ is a continuous function on $[0,1]$ we have

$$
|(T(1))(x)| \equiv 0 \quad \text { or } \quad|(T(1))(x)| \equiv 1 .
$$

Now $|(T(1))(x)| \equiv 0$ implies that $(T(1))(x) \equiv(T(1))^{\prime}(x) \equiv(T(1))^{\prime \prime}(x) \equiv$ $\cdots \equiv(T(1))^{(n)}(x) \equiv 0$ which contradicts $(4)$.

Hence $|(T(1))(x)| \equiv 1$ from which it follows that $(T(1))^{\prime}(x) \equiv 0$ and hence

$$
T(1) \equiv e^{i \lambda} \quad \text { for some fixed } \lambda \in[-\pi, \pi]
$$

We denote $T^{*}\left(L_{\left(x, \theta_{1}, \ldots, \theta_{n}\right)}\right)$ by

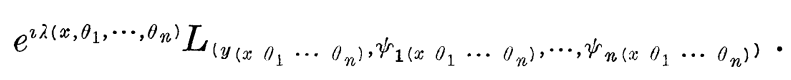

The above Lemma 2.3 , shows that $\lambda\left(x, \theta_{1}, \cdots, \theta_{n}\right) \equiv \lambda$ for $\operatorname{all}\left(\theta_{1}, \cdots, \theta_{n}\right) \in$ $[-\pi, \pi]$. For

$$
\left(T^{*}\left(L_{\left(x, \theta_{1}, \cdots, \theta_{n}\right)}\right)\right)(1)=e^{i \lambda\left(x, \theta_{1}, \cdots, \theta_{n}\right)} L_{\left(y\left(x, \theta_{1}, \ldots, \theta_{n}\right), \psi_{1\left(x, \theta_{1}\right.}, \ldots, \theta_{n}\right) \cdots \gamma_{n}\left(x, \theta_{1}, \ldots, \theta_{n}\right)}(1),
$$

so that $L_{\left(x, \theta_{1}, \cdots, \theta_{n}\right)}(T(1))=e^{i \lambda\left(x, \theta_{1}, \cdots, \theta_{n}\right)}$ and thus $L_{\left(x, \theta_{1}, \cdots, \theta_{n}\right)}\left(e^{i \lambda}\right)=e^{i \lambda\left(x, \theta_{1}, \cdots, \theta_{n}\right)}$. Hence $\lambda\left(x, \theta_{1}, \cdots, \theta_{n}\right) \equiv \lambda$.

Lemma 2.4. If $x \in[0,1]$, then for all $\left(\theta_{1}, \cdots, \theta_{n}\right) \in[-\pi, \pi]^{n}$,

$$
y_{\left(x, \theta_{1}, \cdots, \theta_{n}\right)}=y_{(x, 0, \cdots, 0)} \text {. }
$$

Proof. For fixed $x \in[0,1]$, we consider the map $\rho:[-\pi, \pi]^{n} \rightarrow[0,1]$ given by

$$
\rho\left(\theta_{1}, \theta_{2}, \cdots, \theta_{n}\right)=y_{\left(x, \theta_{1}, \cdots, \theta_{n}\right)} .
$$

It is easy to verify that this mapping is continuous. Hence the image of $[-\pi, \pi]^{n}$ in $[0,1]$ is a connected subset of $[0,1]$. It is, in fact, a singleton. For otherwise we could find $g$ in $C^{(n)}$ such that $g \equiv g^{\prime} \equiv \ldots \equiv g^{(n)} \equiv 0$ on an open subinterval $I \subset \rho\left([-\pi, \pi]^{n}\right)$ while for some $y_{\left(x, \varphi_{1}, \cdots, \varphi_{n}\right)} \notin I$,

$$
\mid g\left(y_{\left(x, \varphi_{1}, \ldots, \varphi_{n}\right)}\right)+e^{\left.i \gamma_{1(x} \varphi_{1}, \ldots, \varphi_{n}\right)} g^{\prime}\left(y_{\left(x, \varphi_{1}, \ldots, \varphi_{n}\right)}\right)
$$




$$
\begin{aligned}
& +e^{i \psi_{2\left(x, \varphi_{1}, \ldots, \varphi_{n}\right)}} \cdot \frac{1}{2 !} g^{\prime \prime}\left(y_{\left(x, \varphi_{1}, \cdots, \varphi_{n}\right)}\right)+\cdots \\
& +e^{i \psi(n-1)\left(x, \varphi_{1}, \ldots, \varphi_{n}\right)} \cdot \frac{1}{(n-1) !} g^{(n-1)}\left(y_{\left(x, \varphi_{1}, \cdots, \varphi_{n}\right)}\right) \mid \\
& <\left|\frac{1}{n !} g^{(n)}\left(y_{\left(x, \varphi_{1}, \ldots, \varphi_{n}\right)}\right)\right|
\end{aligned}
$$

For instance, one may take

$$
g(y)= \begin{cases}0 & y \leqq y_{1} \\ \left(y-y_{1}\right)^{(n+1)} & y>y_{1}\end{cases}
$$

where $y_{1}$ is least upper bound of $I$ and $y_{\left(x, \varphi_{1}, \ldots, \varphi_{n}\right)}$ sufficiently near to $y_{1}$. Thus for an infinite number of $\left(\theta_{1}, \theta_{2}, \cdots, \theta_{n}\right) \in[-\pi, \pi]^{n}$ with $y_{\left(x, \theta_{1}, \cdots, \theta_{n}\right)} \in I$,

$$
\begin{aligned}
L_{\left(x, \theta_{1}, \cdots, \theta_{n}\right)}(T(g)) & =T^{*} L_{\left(x, \theta_{1}, \cdots, \theta_{n}\right)}(g) \\
& =e^{i \lambda} L_{\left(y\left(x, \theta_{1}, \cdots, \theta_{n}\right), \psi_{1\left(x, \theta_{1}\right.}, \cdots, \theta_{n}\right), \cdots, \psi_{n\left(x, \theta_{1}, \cdots, \theta_{n}\right)}(g)} \\
& =0
\end{aligned}
$$

while

$$
\begin{aligned}
& L_{\left(x, \varphi_{1}, \ldots, \varphi_{n}\right)}(T(g)) \\
& \quad=e^{i \lambda} L_{\left(y\left(x, \varphi_{1}, \ldots, \varphi_{n}\right), \psi_{1\left(x, \varphi_{1}\right.}, \ldots, \varphi_{n}\right), \cdots, \psi_{n\left(x, \varphi_{1}, \cdots, \varphi_{n}\right)}}(g) \neq 0 .
\end{aligned}
$$

Since $\rho$ is continuous, $\rho^{-1}(I)$ is open in $[-\pi, \pi]^{n}$ and therefore for each $i=1,2, \cdots, n$ there exist an infinite number of $\theta_{i}$ 's such that

$$
L_{\left(x, \theta_{1}, \ldots, \theta_{n}\right)}(T(g))=0 \text { while } L_{\left(x, \varphi_{1}, \ldots, \varphi_{n}\right)}(T(g)) \neq 0 .
$$

Therefore $(T(g))(x)+e^{i \theta_{1}}(T(g))^{\prime}(x)+\cdots+\left(e^{i \theta_{n}} / n !\right)(T(g))^{(n)}(x)=0$.

For any $j$ with $1 \leqq j \leqq n$, by keeping $\theta_{i}$ constant for $i \neq j$ and varying $\theta_{j}$ we can see that $(T(g))^{(j)}(x)=0$. Thus $L_{\left(x, \varphi_{1}, \cdots, \varphi_{n}\right)}(T(g))=0$ which contradicts (5).

Hence $y_{\left(x, \theta_{1}, \cdots, \theta_{n}\right)}=y_{(x, 0, \cdots, 0)}$ for all $\left(\theta_{1}, \cdots, \theta_{n}\right) \in[-\pi, \pi]^{n}$.

Finally, we define a point map $\tau$ of $[0,1]$ to $[0,1]$ by

$$
\tau(x)=y_{(x, 0, \cdots, 0)} \text {. }
$$

Consideration of $\left(T^{-1}\right)^{*}$ shows that $\tau$ is onto, and, applying Lemma 2.4, one-one.

THEOREM 2.5. Let $T$ be an isometry of $C^{(n)}$. Then, for $f \in C^{(n)}$,

$$
(T(f))(x)=e^{i \lambda} f(\tau(x))
$$

with $e^{i \lambda}=T(1) . \quad M o r e o v e r, ~ \tau$ is one of the two functions $F, 1-F$ where $F$ is the identity mapping of $[0,1]$ onto itself. 
Proof. Given $x \in[0,1]$ and $\theta \in[-\pi, \pi]$, consider the function $g$ of the Proposition 1.1 constructed for $(x, \theta, \cdots, \theta)$. Clearly, $g$ does not depend on $\theta ; g(x)=0 ; g^{\prime}(x), g^{\prime \prime}(x), \cdots, g^{(n)}(x)$ are positive reals and $\sum_{r=1}^{n}\left(g^{(r)}(x) / r !\right)>\sum_{r=0}^{n}\left(\left|g^{(r)}(y)\right| / r !\right)$ for all $y \in[0,1], y \neq x$. Therefore,

$$
\begin{aligned}
\|g\| & =g^{\prime}(x)+\frac{1}{2 !} g^{\prime \prime}(x)+\cdots+\frac{1}{n !} g^{(n)}(x) \\
& =e^{-i \theta} L_{(x, \theta, \cdots, \theta)}(g) \\
& =e^{-i \theta} T^{*} L_{(x, \theta, \cdots, \theta)}\left(T^{-1}(g)\right) \\
& =e^{i(\lambda-\theta)} L_{\left(\tau(x), \psi_{1(x}, \ldots, \theta\right)}, \cdots, \psi_{n(x, \theta, \cdots, \theta)}\left(T^{-1}(g)\right) .
\end{aligned}
$$

Thus we have for all $\theta \in[-\pi, \pi]$

$$
\begin{aligned}
\|g\|= & e^{i(\lambda-\theta)}\left[\left(T^{-1}(g)\right)(\tau(x))+e^{i \psi_{1}(x \theta, \cdots, \theta)}\left(T^{-1}(g)\right)^{\prime}(\tau(x))\right. \\
& \left.+\cdots+\frac{1}{n !} e^{i \gamma_{n}\langle x \theta, \cdots \theta)}\left(T^{-1}(g)\right)^{(n)}(\tau(x))\right]
\end{aligned}
$$

Since

$$
\begin{aligned}
\|g\| & =\left\|T^{-1}(g)\right\| \\
& =\operatorname{Sup}_{y \in[0,1]} \sum_{r=0}^{n} \frac{\left|\left(T^{-1}(g)\right)^{(r)}(y)\right|}{r !},
\end{aligned}
$$

by (6) we have

$$
\|g\|=\left|\left(T^{-1}(g)\right)(\tau(x))\right|+\left|\left(T^{-1}(g)\right)^{\prime}(\tau(x))\right|+\cdots+\frac{1}{n !}\left|\left(T^{-1}(g)\right)^{(n)}(\tau(x))\right| .
$$

Again since $g$ is independent of $\theta$,

$$
\left(T^{-1}(g)\right)(\tau(x)),\left(T^{-1}(g)\right)^{\prime}(\tau(x)), \cdots,\left(T^{-1}(g)\right)^{(n)}(\tau(x))
$$

are independent of $\theta$ but

$$
A(\theta)=\left\{e^{i \psi_{1}(x, 0, \cdots, 0)}\left(T^{-1}(g)\right)^{\prime}(\tau(x))+\cdots+\frac{1}{n !} e^{i \psi_{n}(x, \theta, \cdots, 0)}\left(T^{-1}(g)\right)^{(n)}(\tau(x))\right\}
$$

depends on $\theta$ for otherwise (6) cannot be true. In other words, $A(\theta)$ is not constant. Now by (6) $A(\theta)$ must be on a circle with center as $\left\{-\left(T^{-1}(g)\right)(\tau(x))\right\}$ and radius equal to $\|g\|$.

On the other hand $A(\theta)$ must be on or within the circle with center as origin and radius equal to $\rho=\sum_{r=1}^{n}\left(\left|\left(T^{-1}(g)\right)^{(r)}(x)\right| / r !\right)=\|g\|-$ $\left|\left(T^{-1}(g)\right)(\tau(x))\right|$. This implies that $\left(T^{-1}(g)\right)(\tau(x))=0$ for otherwise $A(\theta)$ has to be a constant (see Figure 2.1) which is false. 


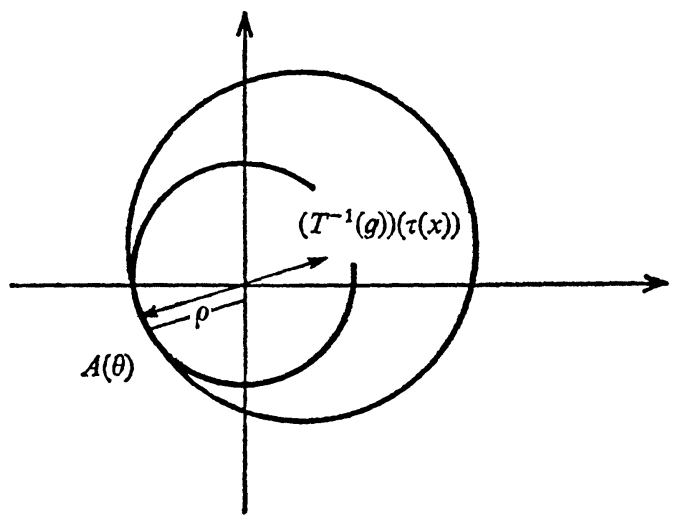

Figure 2.1.

Therefore, we have

$$
\begin{aligned}
\arg e^{i \psi_{1}(x, \theta, \ldots, \theta)}\left(T^{-1}(g)\right)^{\prime}(\tau(x)) & =\arg \cdot \frac{1}{2 !} e^{i \psi_{2}(x, \theta, \cdots, \theta)}\left(T^{-1}(g)\right)^{\prime \prime}(\tau(x))=\cdots \\
& =\arg \cdot \frac{1}{n !} e^{i \psi_{n}(x, \theta, \cdots, \theta)}\left(T^{-1}(g)\right)^{(n)}(\tau(x)) .
\end{aligned}
$$

Thus for all $\theta \in[-\pi, \pi], 1 \leqq k \leqq n, 1 \leqq j \leqq n$

$$
\psi_{k(x, \theta, \cdots, \theta)}-\psi_{j(x, \theta, \cdots, \theta)}=\psi_{k(x, 0, \cdots, 0)}-\psi_{j(x, 0, \cdots, 0)} .
$$

Also by (6)

$$
\begin{aligned}
\|g\| & =e^{i(\lambda-\theta)}\left[\sum_{k=1}^{n} \frac{1}{k !} e^{i \psi^{\prime} k(x, \theta, \cdots, \theta)}\left(T^{-1}(g)\right)^{(k)}(\tau(x))\right] \\
& =e^{i\left(\lambda-\theta+\psi_{j}(x, \theta, \ldots, \theta)\right)}\left[\sum_{k=1}^{n} \frac{1}{k !} e^{i\left(\psi_{k}(x, \theta, \cdots, \theta)-\psi_{j}(x, \theta, \cdots, \theta)\right.}\left(T^{-1}(g)\right)^{(k)}(\tau(x))\right] \\
& =e^{i\left(\lambda-\theta+\psi_{j}(x, \theta, \cdots, \theta)\right.}\left[\sum_{k=1}^{n} \frac{1}{k !} e^{i\left(\psi_{k(x, 0, \cdots, 0)-\psi_{j}(x, 0, \cdots, 0)}\left(T^{-1}(g)\right)^{(k)}(\tau(x))\right.}\right] .
\end{aligned}
$$

Since the left hand side is independent of $\theta$, we have

$$
\left.\lambda-\theta+\psi_{j(x, \theta, \cdots, \theta)}=\lambda+\psi_{j(x, 0, \cdots, 0)}\right) .
$$

Hence for all $\theta \in[-\pi, \pi], 1 \leqq j \leqq n$

$$
\psi_{j(x, \theta, \cdots, \theta)}=\psi_{j(x, 0, \cdots, 0)}+\theta \text {. }
$$

Now let $f$ be any element of $C^{(n)}$ such that $f(x)=0$ then for all $\theta \in[-\pi, \pi]$

$$
f^{\prime}(x)+\frac{1}{2 !} f^{\prime \prime}(x)+\cdots+\frac{1}{n !} f^{(n)}(x)
$$




$$
\begin{aligned}
& =e^{-i \theta} L_{(x, \theta, \cdots, \theta)}(f) \\
& =e^{-i \theta} T^{*} L_{\langle x, \theta, \cdots, \theta)}\left(T^{-1}(f)\right) \\
& =e^{i(\lambda-\theta)} L_{\left\langle\tau(x), \psi_{1(x, \theta,}, \cdots, \theta\right), \cdots \gamma_{n}(x, \theta, \cdots, \theta)}\left(T^{-1}(f)\right) \\
& =e^{i(\lambda-\theta)}\left[\left(T^{-1}(f)\right)(\tau(x))+\sum_{k=1}^{n} \frac{1}{k !} e^{i \psi_{k}(x, \theta, \cdots, \theta)}\left(T^{-1}(f)\right)^{(k)}(\tau(x))\right] \\
& =e^{i \lambda}\left[e^{-i \theta}\left(T^{-1}(f)\right)(\tau(x))+\sum_{k=1}^{n} \frac{1}{k !} e^{\left.i \psi_{k(x, 0, \cdots, 0)}\left(T^{-1}(f)\right)^{(k)}(\tau(x))\right]}\right.
\end{aligned}
$$

so that $\left(T^{-1}(f)\right)(\tau(x))=0$. For an arbitrary $f \in C^{(n)}$, define $g(y)=$ $f(y)-f(x), y \in[0,1]$ then $g(x)=0$ and so

$$
\begin{aligned}
0=\left(T^{-1}(g)\right)(\tau(x)) & =\left(T^{-1}(f)\right)(\tau(x))-f(x)\left(T^{-1}(1)\right)(\tau(x)) \\
& =\left(T^{-1}(f)\right)(\tau(x))-e^{-i \lambda} f(x) .
\end{aligned}
$$

Thus, replacing $f$ by $T(f)$, it follows that for all $x \in[0,1]$ and $f \in C^{(n)}$,

$$
(T(f))(x)=e^{i \lambda} f(\tau(x)) .
$$

Now if, for $0 \leqq v \leqq n-1, F_{r}$ is the mapping of $[0,1]$ onto itself given by $F_{r}(x)=x^{r+1}$ (where $F_{0}$ is the identity map $F$ ), we have

$$
\left(T\left(F_{r}\right)\right)(x)=e^{i \lambda}(\tau(x))^{r+1}=e^{i \lambda}\left(\tau^{r+1}\right)(x), \quad 0 \leqq r \leqq n-1 .
$$

Therefore $\left(T\left(F_{r}\right)\right)(x)=\left(T\left(F_{r-1}\right)\right)(x) \cdot \tau(x)$. Now

$$
\begin{aligned}
\sum_{k=0}^{n} \frac{1}{k !}\left(T\left(F_{r}\right)\right)^{(k)}(x) & =L_{(x, 0, \cdots, 0)}\left(T\left(F_{r}\right)\right) \\
& =T^{*} L_{(x, 0, \cdots, 0)}\left(F_{r}\right) \\
& =e^{i \lambda} L_{\left(\tau(x), \psi_{1(x, 0, \cdots, 0)}, \ldots, \psi_{n(x, 0, \cdots, 0)}\left(F_{r}\right)\right.} \\
& =e^{i \lambda}\left[F_{r}(\tau(x))+\sum_{j=1}^{n} \frac{1}{j !} e^{i \psi_{j}(x, 0, \cdots, 0)} F_{r}^{(j)}(\tau(x))\right] \\
& =e^{i \lambda}\left[(\tau(x))^{r+1}+\sum_{j=1}^{r+1} e^{i \psi_{j}(x, 0, \cdots, 0)} C_{j}^{r+1}(\tau(x))^{r+1-j}\right] .
\end{aligned}
$$

Thus for $0 \leqq r \leqq n-1$

$$
\sum_{k=1}^{n} \frac{1}{k !}\left(T\left(F_{r}\right)\right)^{(k)}(x)=e^{i \lambda} \sum_{j=1}^{r+1} e^{i \psi_{j}(x 0, \cdots, 0)} C_{j}^{r+1}(\tau(x))^{r+1-j} .
$$

Taking $r=0$ in (7), we get

$$
\sum_{k=1}^{n} \frac{1}{k !}(T(F))^{(k)}(x)=e^{i\left(\lambda+\psi_{1}(x, 0, \cdots, 0)\right)} .
$$

Taking $r=1$, we get 


$$
\sum_{r=1}^{n} \frac{1}{k !}\left(T\left(F_{1}\right)\right)^{(k)}(x)=C_{1}^{2}(\tau(x)) e^{i\left(\lambda+\psi_{1}(x, 0, \cdots, 0)\right)}+e^{i\left(\lambda+\psi_{2}(x, 0, \cdots, 0)\right)}
$$

Hence

$$
\begin{aligned}
& e^{i\left(\lambda+\psi_{2}(x, 0, \cdots, 0)\right)} \\
& \quad=\sum_{k=1}^{n} \frac{1}{k !}\left(T\left(F_{1}\right)\right)^{(k)}(x)-C_{1}^{2}(\tau(x)) \sum_{k=1}^{n} \frac{1}{k !}(T(F))^{(k)}(x) .
\end{aligned}
$$

Thus by successive iterations we get for $1 \leqq r \leqq n$

$$
\begin{aligned}
e^{i\left(\lambda+\psi_{r}(x, 0, \ldots, 0)\right)} & =\sum_{k=1}^{n} \frac{1}{k !}\left\{\sum_{j=1}^{r}(-1)^{j-1} C_{j-1}^{r}\left(T\left(F_{r-j}\right)\right)^{(k)}(x)(\tau(x))^{j-1}\right\} \\
& =e^{i \lambda} \sum_{k=1}^{n} \frac{1}{k !}\left\{\sum_{j=1}^{r}(-1)^{j-1} C_{j-1}^{r}\left(\tau^{r-j+1}\right)^{(k)}(x)(\tau(x))^{j-1}\right\} .
\end{aligned}
$$

Therefore,

$$
e^{\left.i \psi_{n(x, 0}, \cdots, 0\right)}=\sum_{k=1}^{n} \frac{1}{k !}\left\{\sum_{j=1}^{n}(-1)^{j-1} C_{j-1}^{n}\left(\tau^{n-j+1}\right)^{(k)}(x)(\tau(x))^{j-1}\right\} .
$$

Applying Proposition 1.2 to the function $\tau$ which clearly belongs to $C^{(n)}$ we get

$$
e^{i \psi_{n}(x, 0, \cdots, 0)}=\left\{\tau^{\prime}(x)\right\}^{n} .
$$

Thus $\tau^{\prime}(x)$ is an $n$th root of a complex number of absolute value one.

But since $\tau^{\prime}(x)$ is real valued and continuous we have $\tau^{\prime}(x) \equiv 1$ or $\tau^{\prime}(x) \equiv-1$ and, therefore, $\tau(x) \equiv F$ or $1-F$.

\section{REFERENCES}

1. M. Cambern, Isometries of certain Banach algebras, Studia Mathematica T., XXV , (1965), 217-225.

2. N. V. Rao and A. K. Roy, Linear isometries of some function spaces, Pacific J. Math., 38 (1971), 177-192.

Received February 2, 1979 and in revised form April 27, 1979.

UNIVERSITY OF CALIFORNIA

Santa Barbara, CA 93106

Current address: M. S. University

Baroda-390 002

India 


\section{PACIFIC JOURNAL OF MATHEMATICS}

\section{EDITORS}

DONALD BABBITT (Managing Editor)

University of Galifornia

Los Angeles, California 90024

Hugo RossI

University of Utah

Salt Lake City, UT 84112

C. C. MOORE AND ANDREW OGG

University of California

Berkeley, CA 94720

\section{J. DugundjI}

Department of Mathematics University of Southern California

Los Angeles, California 90007

R. Finn AND J. Milgram

Stanford University

Stanford, California 94305

\section{ASSOCIATE EDITORS}

R. ARENS

E. F. BECKENBACH

B. H. NeUmanN

F. WOLF

K. YosHIDA

\section{SUPPORTING INSTITUTIONS}

UNIVERSITY OF ARIZONA

UNIVERSITY OF BRITISH COLUMBIA

CALIFORNIA INSTITUTE OF TECHNOLOGY

UNIVERSITY OF CALIFORNIA

MONTANA STATE UNIVERSITY

UNIVERSITY OF NEVADA, RENO

NEW MEXICO STATE UNIVERSITY

OREGON STATE UNIVERSITY
UNIVERSITY OF OREGON

UNIVERSITY OF SOUTHERN CALIFONIA

STANFORD UNIVERSITY

UNIVERSITY OF HAWAII

UNIVERSITY OF TOKYO

UNIVERSITY OF UTAH

WASHINGTON STATE UNIVERSITY

UNIVERSITY OF WASHINGTON 


\section{Pacific Journal of Mathematics}

\section{Vol. 94, No. 1 \\ May, 1981}

Willy Brandal, Conditions for being an FGC domain $\ldots \ldots \ldots \ldots \ldots \ldots \ldots$

Allan Calder and Frank Williams, Incompressibility of maps and the

homotopy invariance of Čech cohomology $\ldots \ldots \ldots \ldots \ldots \ldots \ldots \ldots \ldots$

Jacques Chaumat, Quelques propriétés du couple d'espaces vectoriels

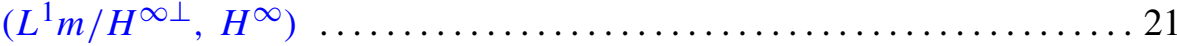

Manfred Droste and Rüdiger Göbel, Products of conjugate permutations . . 47

Jean Esterle, Rates of decrease of sequences of powers in commutative

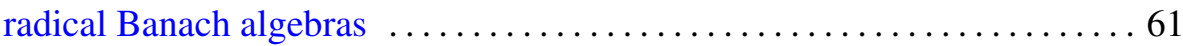

Allan Fryant, Ultraspherical expansions and pseudo analytic functions . . . 83

John Hannah, Homogenization of regular rings of bounded index. II . . . . . 107

Shigeru Haruki, On the theorem of S. Kakutani-M. Nagumo and J. L. Walsh

for the mean value property of harmonic and complex polynomials $\ldots .113$

Hugh M. Hilden, Representations of homology 3-spheres $\ldots \ldots \ldots \ldots \ldots 125$

Craig Huneke, A characterization of locally Macaulay completions . . . . . 131

Takesi Isiwata, Closed ultrafilters and realcompactness ................. 139

Joseph Weston Kitchen, Jr. and David A. Robbins, Tensor products of

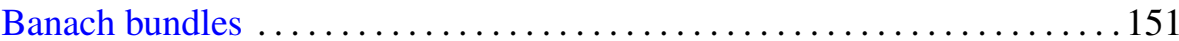

Allan J. Kroopnick, Note on bounded $L^{p}$-solutions of a generalized Liénard

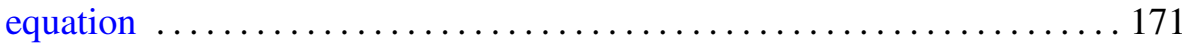

Ajay Kumar and Ajit Kaur Chilana, Spectral synthesis in products and quotients of hypergroups

Charles Livingston, Homology cobordisms of 3-manifolds, knot

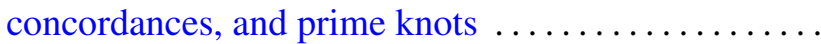

Hans Opolka, Projective representations of finite groups in cyclotomic fields

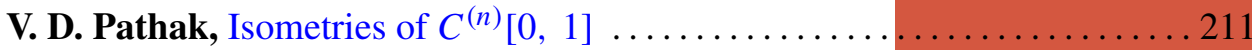

Mark Allan Pinsky, On the spectrum of Cartan-Hadamard manifolds . . . . . 2223

Judith Roitman, The number of automorphisms of an atomic Boolean

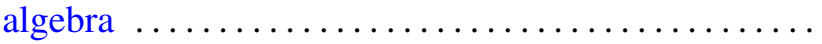

Kai Wang, Locally smooth torus group actions on integral cohomology complex projective spaces 